\title{
GT2020-14463
}

\section{LARGE EDDY SIMULATIONS OF HIGH ROSSBY NUMBER FLOW IN THE HIGH PRESSURE COMPRESSOR INTER-DISK CAVITY}

\author{
Deepak Saini; Richard D. Sandberg \\ Department of Mechanical Engineering \\ University of Melbourne \\ Melbourne, VIC 3010, Australia \\ Email: dsaini@student.unimelb.edu.au
}

\section{ABSTRACT}

The focus of the present study is to understand the effect of Rayleigh number on a high Rossby number flow in a high pressure compressor (HPC) inter-disk cavity. These cavities form between the compressor disks of a gas turbine engine, and they are an integral part of the internal air cooling system. We perform highly resolved large eddy simulations for two Rayleigh numbers of $0.76 \times 10^{8}$ and $1.54 \times 10^{8}$ at a fixed Rossby number of 4.5 by solving the compressible Navier-Stokes equations.

The results show a flow structure dominated by a toroidal vortex in the inner region of the cavity. In the outer region, the flow is observed to move radially outwards by Ekman layers formed on the side disks and to move radially inwards through the central core region of the cavity. An enhancement in the intensity of the radial flares is observed in the outer region of the cavity for the high Rayleigh number case with no perceivable effect in the inner region. The near shroud region is mostly dominated by the centrifugal buoyancy-induced flow and the wall Nusselt number calculated at the shroud is in close agreement with centrifugal buoyancy-induced flow without an axial bore flow.

\section{NOMENCLATURE}

$c_{p}$

$\delta$

LES

$\mu$

$\Omega$

$p$

$r$

$\rho$

RANS specific heat at constant pressure

Ekman depth

axial gap between the disks

Large eddy simulations

dynamic viscosity

angular velocity

pressure

radial direction or radius

density

Reynolds Averaged Navier-Stokes
$S$

$T$

TKE

$\theta$

$u, v, w$

$U_{b u l k}$

$z$

Dimensionless Numbers

$\gamma$

$M a$

$\mathrm{Nu}$

$\mathrm{Pr}$

$R a$

Re

Ro

\section{Subscripts and Superscripts} c

$E k$

$i$

in

$\infty$

$m$

out

O

rms

$S$

sh

$w$

z

$-$

*Address all correspondence to this author.

Sutherland temperature

temperature

turbulent kinetic energy

circumferential direction

velocity in $z, r$ and $\theta$ direction

bulk velocity of axial bore flow

axial direction or axial length

Ratio of specific heats

Mach number

Nusselt number

Prandtl number

Rayleigh number

Reynolds number

Rossby number

value in the core of the cavity

Ekman layer

value at the inner radius of the disk

inlet section of the bore region reference quantity

middle section of the bore region

outlet section of the bore region value at the outer radius of the cavity

root mean square

value at outer wall of the shaft

at the shroud

at the wall

in the axial direction

time averaged

in the wall units 


\section{Introduction}

The evolution of gas turbine technology for future commercial aviation is mainly focused on improving the thermodynamic efficiency, reducing the weight and enhancing the safety of an engine. The theoretical limit of thermodynamic efficiency of an idealized gas turbine cycle is estimated to be $80 \%$ and since the invention of the first working engine the efficiency has increased from $10 \%$ to $50 \%$ [1]. The thermodynamic efficiency of a gas turbine can be further improved by increasing the overall pressure ratio of an engine cycle. However, such change in the operating conditions of the engine would reduce the blade size at the rear end of the compressor and requires precise control of the clearance between the blade tip and the casing of the engine. Besides, it also poses challenges of cooling of the compressor blades due to the average rise in the temperature of the air inside the compressor. In a gas turbine engine, a secondary internal airflow bled from the primary flow path of the compressor is mainly responsible for addressing these challenges. One of the vital functions of the secondary internal air path is to remove excessive heat from the compressor that is detrimental for its safe operation. Figure 1 shows a schematic of the internal air cooling section of the high pressure compressor. The cavities form between the compressor disks of an engine, which is feed by axial throughflow of cold air. The fluid flow and heat transfer inside these annular cavities directly influence the cooling of the compressor blades and thermal expansion of the disks, which controls the blade-tip clearance. Thus, it is crucial to accurately predict the flow physics and induced wall heat transfer rate inside these cavities to optimize the overall design and enhance the safety of an engine.

An initial experimental study on heated side disks was conducted by Farthing et al. [2] [3] to characterize the flow structure and heat transfer rate. They carried out experiments for a cavity with an unheated shroud (top surface of the cavity shown in figure 1) and imposed a radially decreasing temperature distribution on the side disks. A flow structure was proposed shown in figure 2. They observed a radial arm that formed inside the cavity which bifurcated to cyclonic and anti-cyclonic pair of vortices. They speculated that radial inflow occurs inside the boundary layers formed on the side disks. Additional experiments were carried out with insulated cobs (bottom section of the disk shown in figure 1) and two or three radial arms were observed inside the cavity, unlike the one radial arm observed for the cavity without any cobs. In their companion study [3], heat transfer results were presented. A radially increasing Nusselt number profile was reported for the cavity with radially increasing temperature distribution on the side disk. Another experimental study was carried out by Bohn et al. [4] to characterize the flow structure for radially increasing temperature distribution on the side disks. In their experiments, the fluid flow inside the cavity, without cobs and unheated shroud surface, was visualized by using a laser light sheet technique. A flow structure similar to [2] was reported in their study for the radially increasing temperature profiles on the side disk. However, in both the studies [2] [4] the shroud surface was unheated, and the side disks were designed without any cobs. Both these conditions are necessary for the experimental results to be applicable to a real engine cavity.

Thus, to design an experiment more closely representing real engine conditions, an experimental rig for a single cavity with cobs was set up by Owen et al. [5]. The velocity and heat transfer measurements were carried out for one heated disk, and all other surfaces were insulated. The measurement of circumferential velocity showed that the fluid in the core of the cavity rotates at a speed slower than the local disk speed and it further reduced with an increase in the temperature difference. To provide more insights on the experimental results of [5], a subsequent numerical study was carried out by Owen et al. [6] by solving the compressible unsteady RANS equations with a $\kappa-\varepsilon$ turbulence model. A flow structure analogous to previous studies [2] [4] was reported with qualitative agreement with the experiments on the radial distribution of Nusselt number.

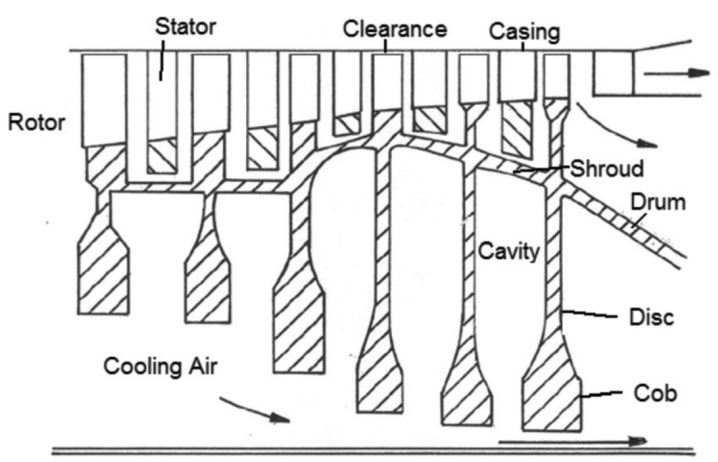

Shaft

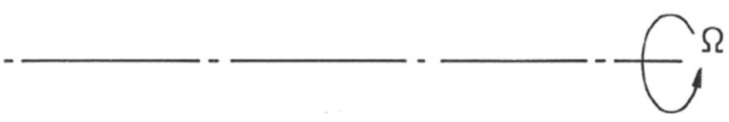

FIGURE 1: Schematic of the internal air cooling path of the high pressure compressor of a gas turbine engine showing the interdisc cavities [7].
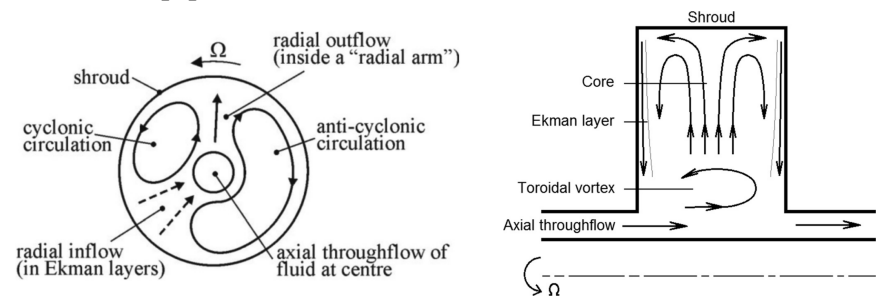

FIGURE 2: Schematic of flow strucutre proposed by Farthing et al. [2] from [7] (Left figure). Schematic of assumed flow strucutre by [8] (Right figure)

Further, to replicate the design architecture of the internal air path, an experimental study on a rig with multiple cavities was conducted by Long et al. [9]. The velocity measurements were carried out for radially increasing temperature distribution on the side disks with a heated shroud. The variation of the circumferential velocity component showed a peak in the inner part of the cavity and decreased to solid-body rotation in the outer part. Based on these measurements, the radial extent of the toroidal vortex was reported to be $r \approx 0.6$. No measurements were carried out inside the disk boundary layers due to the flow unsteadiness. Thus, it was challenging to interpret the overall flow structure, as concluded by the authors. A numerical study was carried out 
by Sun et al. [10] for a cavity configuration matching the experiments of [9] to overcome the limitations of experimental studies. Both LES and URANS calculations were carried out and compared against the experimental data. A strong unsteadiness in the flow structure was observed, and LES results were reported to be in better agreement with experiments. However, no detailed information about the flow structure was provided in their study.

To isolate the effects of axial Reynolds number, rotational Reynolds number and Rayleigh number on the disk temperature profiles, an experimental study was conducted by Atkins and Kanjirakkad [11] on the muti cavity rig of [9]. The experiments were performed for high Rossby number and low Rossby number conditions. In both situations, the Rayleigh number was varied to study its effect on the disk temperature distribution. A negligible effect of Rayleigh number on temperature distribution in the inner region of the cavity was reported with a strong dependence on axial Reynolds number. At low rotational Reynolds number, the mixing level between the axial bore flow and the outer cavity flow was increased as compared to the high rotational Reynolds number, which suppressed the mixing. Further, a study was carried out by Puttock et al. [12] to understand the dependence of shroud heat transfer rate on the axial Reynolds number. The shroud Nusselt number was reported to be dependent only on the shroud Rayleigh number with no discernible effect of axial Reynolds number. The Nusselt number values were in accord with the correlation for turbulent free convection from a flat plate. Further, 3D unsteady RANS calculations were conducted to support the experimental results and showed the existence of Rayleigh-Bénard streaks along the shroud. Further, a pioneering study by Owen and Tang [8] proposed a theoretical model based on the solution of Ekman-layer equations. They assumed a flow structure as shown in figure 2 and assumed a laminar, inviscid and adiabatic core to find the approximate solution for the equations. The final solution showed that the local Nusselt number based on the core temperature is proportional to the core Grashof number to the power of one fourth. Very recent large-eddy simulation results were reported by Pitz et al. [13] to study the effect of axial throughflow on the centrifugal-buoyancy induced flow. The study was conducted on a closed cavity configuration extended to study the effect of bore flow. Thus, the flow features were also compared with the closed cavity results to quantify the effect of bore flow. They carried out the simulations for two axial Reynolds numbers of 1000 and 2000 at a fixed Rayleigh number of $10^{8}$. The thickness of the disk boundary layer was found to be consistent with a laminar Ekman layer, and flow in the core region was observed to be turbulent. The thickness of the kinetic boundary layer formed on the side disks was observed to be varying in the radial direction. However, the authors did not provide any information about the flow structure in the cavity with heated disks and a shroud.

Clearly, there is no general interpretation of the flow structure that develops in a cavity for heated disks with radially increasing temperature profiles and a heated shroud. This is mainly due to the difficulty of the experiments to measure and visualize the fluid flow inside the cavity due to the flow unsteadiness [9]. To our knowledge, there is no highly resolved numerical study which has addressed this problem. Hence, in this study, we carry out highly resolved large eddy simulation for the high Rossby number experimental conditions of [11] to understand the over- all flow structure and study the effect of Rayleigh number.

\section{Problem Description and Numerical Setup}

The computational domain is an annular rotating cavity with an axial bore flow (concentric pipe flow region) that rotates at a constant angular velocity of $\Omega$ about its axis. It comprises of five blocks shown in figure 3 . The inner and outer radius of the bore flow region is $r_{s}, r_{i}$ respectively and $r_{o}$ is the radius of the shroud surface. In the axial direction, $z_{\text {in }}, z_{\text {out }}$ and $z_{m}$ are the domain lengths of the bore flow inlet, outlet and middle sections, respectively, shown in figure 3 . The outer cavity is enclosed by two side disks, spaced $H$ apart. A radially increasing temperature profile is prescribed on the side disks extracted from the experiments by [11], as shown in figure 4 (a) and also provided in the appendix. The temperature is maximum at the shroud $\left(T_{s h}\right)$ and minimum at the outer wall of the shaft $\left(T_{S}\right)$, which is considered as the reference temperature $\left(T_{\infty}=T_{s}\right)$. The outer wall of the bore flow is at temperature $T_{i}>T_{s}$. The cavity is filled with a perfect gas with specific heat at constant pressure $c_{p}$ and ratio of specific heats $\gamma$. At $T_{\infty}$, the dynamic viscosity is $\mu_{\infty}$ and the thermal conductivity is $k_{\infty}$. The compressible NavierStokes equations for the conservative variables are solved in a fixed frame, where the mass, momentum and energy equations, non-dimensionalised with reference velocity $\Omega r_{o}$, length $r_{o}$, density $\rho_{\infty}$ and pressure $\rho_{\infty}\left(\Omega r_{o}\right)^{2}$, are given as

$$
\begin{aligned}
& \frac{\partial \rho}{\partial t}+\frac{\partial}{\partial x_{k}}\left(\rho u_{k}\right)=0, \\
& \frac{\partial}{\partial t}\left(\rho u_{i}\right)+\frac{\partial}{\partial x_{k}}\left[\rho u_{i} u_{k}+p \delta_{i k}-\tau_{i k}\right]=0, \\
& \frac{\partial}{\partial t}(\rho E)+\frac{\partial}{\partial x_{k}}\left[u_{k}(\rho E+p)+q_{k}-u_{i} \tau_{i k}\right]=0,
\end{aligned}
$$

respectively, where the total energy, $E=T /\left[\gamma(\gamma-1) M a_{\infty}^{2}\right]+$ $(1 / 2) u_{i} u_{i}$ and the equation of state is $p=\rho T /\left(\gamma M a_{\infty}^{2}\right)$. The viscous stress tensor and the heat-flux vector are computed as

$$
\begin{aligned}
\tau_{i k} & =\frac{\mu}{R e_{\infty}}\left(\frac{\partial u_{i}}{\partial x_{k}}+\frac{\partial u_{k}}{\partial x_{i}}-\frac{2}{3} \frac{\partial u_{j}}{\partial x_{j}} \delta_{i k}\right), \\
q_{k} & =\frac{-\mu}{(\gamma-1) M a_{\infty}^{2} P r_{\infty} R e_{\infty}} \frac{\partial T}{\partial x_{k}} .
\end{aligned}
$$

The molecular viscosity is computed using the Sutherland law, $\mu=T^{3 / 2}\left(1+S / T_{\infty}\right) /\left(T+S / T_{\infty}\right)$, where $S$ is the Sutherland temperature. The non-dimensional numbers that completely specify this problem are given as

$$
\begin{aligned}
& \operatorname{Re}_{\infty} \equiv \frac{\rho_{\infty}\left(\Omega r_{o}\right) r_{o}}{\mu_{\infty}}, \quad R e_{z} \equiv \frac{\rho_{\infty} U_{\text {bulk }}\left(2\left(r_{i}-r_{s}\right)\right)}{\mu_{\infty}}, \quad \frac{S}{T_{\infty}}=0.37, \\
& \operatorname{Pr}_{\infty} \equiv \frac{\mu_{\infty} c_{p}}{k_{\infty}}=0.71, \quad M a_{\infty} \equiv \frac{\Omega r_{o}}{\left(c_{p}(\gamma-1) T_{\infty}\right)^{1 / 2}}=0.3, \quad \gamma=1.4, \\
& \frac{\Delta T}{T_{\infty}} \equiv \frac{T_{s h}-T_{s}}{T_{s}}, \quad \frac{r_{s}}{r_{o}}=0.2363, \quad \frac{r_{i}}{r_{o}}=0.318, \quad \frac{H}{r_{o}}=0.195, \\
& \frac{z_{\text {in }}}{r_{o}}=0.82, \quad \frac{z_{m}}{r_{o}}=0.117, \quad \frac{z_{\text {out }}}{r_{o}}=0.305,
\end{aligned}
$$


where $\operatorname{Re}_{\infty}\left(=\operatorname{Re}_{\theta}\right), P r_{\infty}$ and $M a_{\infty}$ are the reference Reynolds number, Prandtl number and Mach number, respectively. The axial Reynolds number $R e_{z}$ is defined based on the bulk velocity $\left(U_{\text {bulk }}\right)$ at the inlet, specified as the boundary condition. A linear temperature profile is specified at the inlet varying from the shaft temperature $T_{\infty}$ to the temperature at the outer wall of bore flow $T_{i}$ as provided by [11]. To further define the relative strength of the axial inertial force with respect to the rotational inertial force, the Rossby number is defined as

$$
R o \equiv \frac{U_{b u l k}}{\Omega r_{o}}=\frac{r_{o}^{2}}{2 r_{i}\left(r_{i}-r_{s}\right)} \frac{R e_{z}}{R e_{\theta}} .
$$

It is important to define the Rayleigh number to quantify the effect of centrifugal induced buoyancy force relative to the viscous force in the flow as

$$
\begin{aligned}
& R a \equiv \frac{\rho_{\infty}^{2} c_{p}\left[\Omega^{2}\left(r_{o}+r_{i}\right) / 2\right]\left(\Delta T / T_{\infty}\right)\left(r_{o}-r_{i}\right)^{3}}{\mu_{\infty} k_{\infty}} \\
& =\operatorname{Re}_{\theta}^{2} \operatorname{Pr}_{\infty} \frac{\Delta T}{T_{\infty}} \frac{1}{2}\left(1+\frac{r_{i}}{r_{o}}\right)\left(1-\frac{r_{i}}{r_{o}}\right)^{3} .
\end{aligned}
$$

The response of the system is the local Nusselt number on the side disks, which is the non-dimensional form of the wall heat transfer coefficient defined as

$$
N u_{r}=\frac{r}{T_{w}-T_{r e f}}\left[-\frac{d \bar{T}}{d z}\right]_{w} .
$$

In cylindrical coordinates, $u, v$ and $w$ represents the nondimensional velocity components in the $z, r$ and $\theta$ directions, respectively. The boundary conditions for this problem are thus $u=v=0$ for all the walls of the cavity. The inner wall of the bore flow region is stationary, $w\left(z, r=r_{s} / r_{o}, \theta\right)=0$, and the outer wall is rotating at constant angular velocity, $w\left(z, r=r_{i} / r_{o}, \theta\right)=r_{i} / r_{o}$. The rotating wall boundary condition for the side disks and shroud is $w(z= \pm H / 2, r, \theta)=r, w(z, r=1, \theta)=1$, respectively. In this study, we are analyzing just one cavity and ignoring the effect of other cavities onto the bore flow conditions. Thus, it is more sensible to have fully developed turbulent flow conditions in the bore flow at the cavity inlet rather than laminar conditions. For that, a precursor periodic concentric pipe flow calculation (Block I in figure 3) is carried out at axial Reynolds number of $R e_{z}=18,405$ to obtain fully developed flow profiles. The turbulence at the bore flow inlet is then generated by a digital filter technique (Touber and Sandham [14]), which uses the mean velocity and Reynolds stress profiles extracted from the precursor simulation and then superposes the fluctuations onto the mean profiles to generate turbulent inlet conditions. The axial domain size of the bore flow inlet $\mathrm{z}_{\text {in }} / r_{0}=0.82$ is found to be sufficient to recover the fully developed turbulent velocity profile and turbulent kinetic energy from the periodic precursor simulation, as shown in figures 4 (b) and 4 (c). The axial domain size of the bore flow outlet (Block III) is $\mathrm{z}_{\text {in }} / r_{0}=0.305$, evaluated by running different length sizes, which was enough to nullify the effect of the outlet boundary condition on the cavity. To further avoid any disturbance from the outlet boundary to move upstream, the zonal non-reflecting characteristic boundary condition (Sandberg and Sandham [15]) is used over the last $\mathrm{z} / r_{o}=0.128$ of the outlet block.

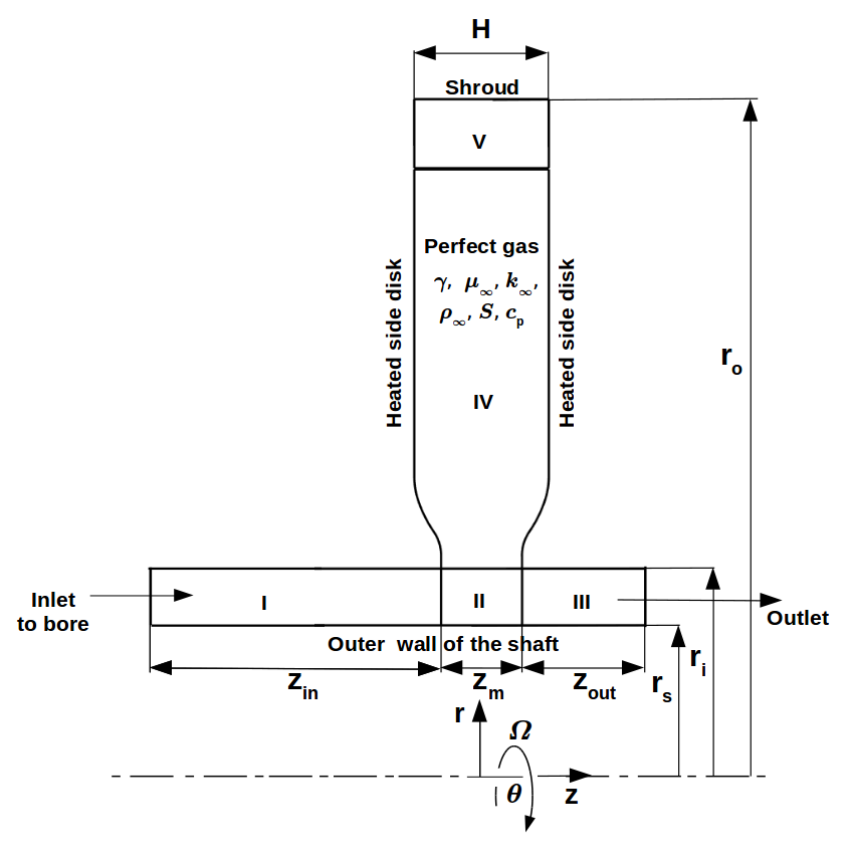

FIGURE 3: Schematic of inter-disk cavity. The walls of the cavity undergo solid-body rotation about the $z$-axis except the shaft, which is held stationary to match the conditions of [11].

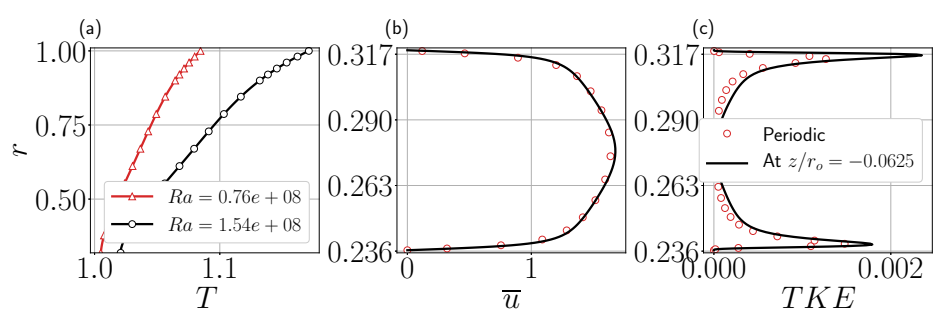

FIGURE 4: (a) Temperature profiles for the side disks from [11], Comparison of fully developed (b) mean axial velocity profile and (c) turbulent kinetic energy (TKE) in the bore region obtained by digital filter inflow at the downstream location $z / r_{o}=$ -0.0625 from the inlet with the profiles from precursor periodic simulation.

The code HiPSTAR (High Performance Solver for Turbulence and Aeroacoutics Research) [16] was used, where the axial $(z)$ and radial $(r)$ directions were discretised with a fourth-order accurate standard finite-difference scheme and the circumferential $(\theta)$ direction was discretised with a Fourier spectral method. Time integration was achieved using a low-storage fourth-order 
accurate Runge-Kutta scheme. To enhance the stability of the code skew-symmetric splitting of the non-linear terms and an 11 point wave-number-optimized filter is applied to the solution after each full time step with low weighting to reduce grid-to-grid point oscillations. The code has been used and validated for a range of turbomachinery applications ( [17] [18] [19]) and more recently for simulating the centrifugal buoyancy-induced flow in a closed cavity [20] [21].

\section{Results and Discussion}

Large eddy simulations have been carried out for two Rayleigh numbers matching the experimental conditions of [11], as shown in Table 2. The number of grid points for each block is also reported in Table 2 .

\section{Grid quality}

In this study, the standard WALE model [22] is used to account for the sub-grid stresses. To assess the influence of the LES model onto the solution, the sub-grid scale viscosity $\mu_{s g s}$ is compared with the molecular viscosity as shown in figure 5. It is less than 1 in most of the cavity except in the small region near the cob, where the maximum value is close 1.3 for both $R a$ numbers. For $R a=1.54 e+08$, the region with the maximum value of $\mu_{s g s} / \mu$ has become larger compared to the low $R a$ number case, but the maximum value remains the same. To further evaluate the grid quality, the sub-grid scale viscosity $\mu_{s g s}$ is also compared with the turbulent eddy viscosity for both cases. The comparison shows that we are resolving $99 \%$ of the turbulent energy with the current grid resolution.

The grid-spacings in wall units for the shroud, upstream disk and downstream disk are shown in figure 6. For both $R a$ numbers, the wall-normal spacing $\Delta r^{+}$is less than 0.05 , and the grid is stretched in the axial direction with maximum grid-spacing $\Delta z^{+}<2$ at the shroud. In the circumferential direction, $r \Delta \theta^{+}$ is less than 5 for both $R a$ numbers at the shroud. Similarly, for the side disks, the wall-normal resolution $\Delta z^{+}$is in most regions less than 0.5 with a maximum value close to 0.7 at the inner region of the downstream disk (figure $6(\mathrm{~h})$ ), where the axial bore flow enters the cavity. The grid is stretched in the radial direction at the side disk with a maximum $\Delta r^{+}<10$ in the middle of the cavity at the downstream disk. The grid resolution in the circumferential direction $r \Delta \theta^{+}$has a maximum value close to 20 near to the cavity inlet at the downstream disk. To further ascertain the adequacy of the grid resolution in the circumferential direction to resolve the turbulent scales, the wavenumber spectra of radial velocity at different radial locations are plotted in figure 7 . The spectra follow the $5 / 3$ power law in the inertial range and a decay of about four orders of magnitude is observed in the amplitude of energy at $r=0.4$ and 0.5 for both the $R a$ numbers. These two numerical probes are placed in the toroidal vortex region, where the overall energy level is high due to the breakdown of the large structures to small scales. However, the decay in the amplitude of energy is increased to six orders of magnitude for the rest of the points in the cavity, where mostly large scale structures are present. The values for all these metrics give us high confidence that the simulations are adequately resolved with the current grid resolution in all directions and that the results are reliable.

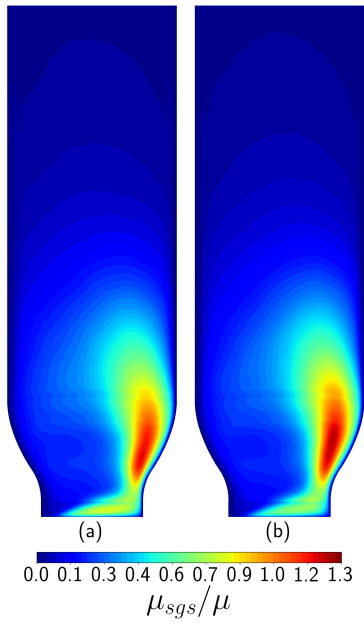

FIGURE 5: Comparison of sub-grid scale viscosity with molecular viscosity for (a) $R a=0.76 e+08$ and (b) $R a=1.54 e+08$.
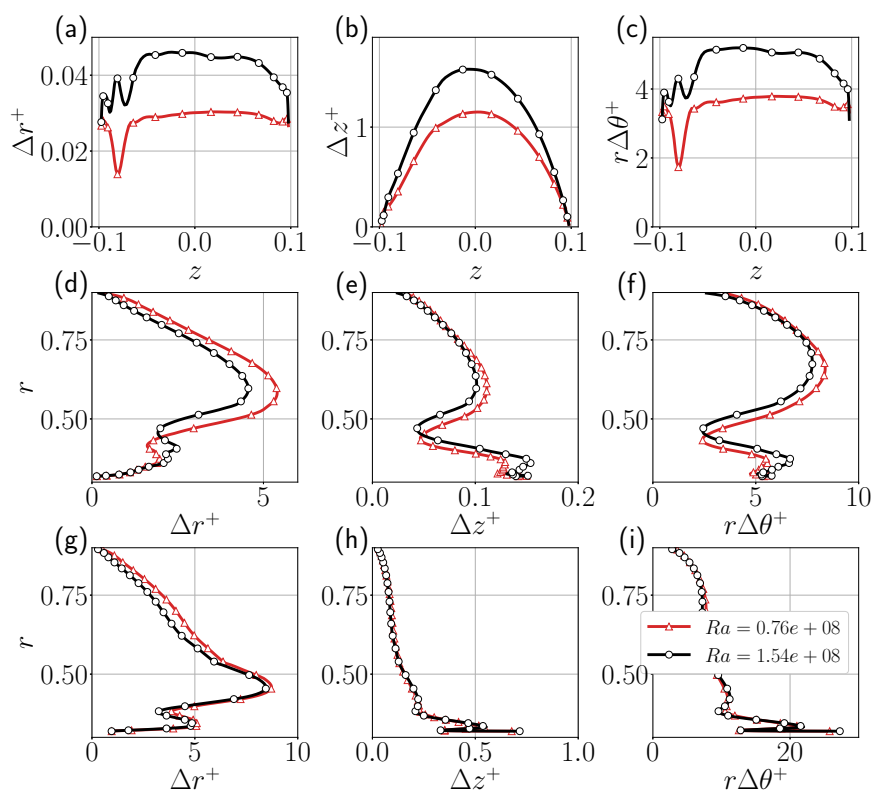

FIGURE 6: Grid-spacing in wall units for the shroud $(a-b)$, upstream disk $(d-f)$ and downstream disk $(g-i)$ at different Rayleigh numbers.

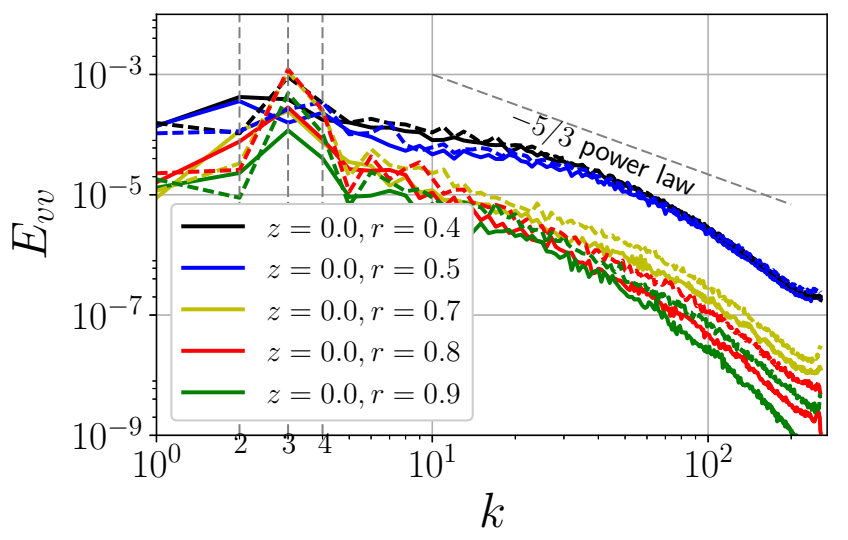

FIGURE 7: Wavenumber spectra of different monitor points located in the cavity at different Rayleigh numbers $(-R a=$ $\left.0.76 \times 10^{8},---R a=1.54 \times 10^{8}\right)$. 
TABLE 1: Simulation Parameters

\begin{tabular}{|c|c|c|}
\hline $\begin{array}{c}\text { Non- } \\
\text { Dimensional } \\
\text { numbers }\end{array}$ & Case I & Case II \\
\hline $\operatorname{Re}_{\theta}$ & 77,900 & 77,900 \\
\hline$R e_{z}$ & 18,405 & 18,405 \\
\hline$M a_{\infty}$ & 0.3 & 0.3 \\
\hline$S / T_{\infty}$ & 0.37159 & 0.37159 \\
\hline Ro & 4.50 & 4.50 \\
\hline$R a$ & $0.76 e+08$ & $1.54 e+08$ \\
\hline$\Delta T / T_{\infty}$ & 0.0846 & 0.1712 \\
\hline$p_{\text {out }}$ & 8.187 & 8.196 \\
\hline Grid Size & $N_{r}, N_{z}, N_{\theta}$ & $N_{r}, N_{z}, N_{\theta}$ \\
\hline Block I & $100,162,514$ & $100,162,514$ \\
\hline Block II & $100,104,514$ & $100,104,514$ \\
\hline Block III & $100,66,514$ & $100,66,514$ \\
\hline Block IV & $192,104,514$ & $208,104,514$ \\
\hline Block V & $108,104,514$ & $152,104,514$ \\
\hline $\begin{array}{l}\text { Dimensional } \\
\text { quantities to } \\
\text { match the } \\
\text { conditions } \\
\text { of [11] }\end{array}$ & Case I & Case II \\
\hline $\begin{array}{c}\text { Bore mass } \\
\text { flow rate } \\
\quad(\mathrm{kg} / \mathrm{s})\end{array}$ & 0.0672 & 0.0672 \\
\hline RPM & 199.0 & 199.0 \\
\hline$T_{\infty}\left(=T_{S}\right)(K)$ & 297.1 & 297.1 \\
\hline$T_{i}(K)$ & 298.99 & 303.26 \\
\hline$r_{o}(m)$ & 0.22 & 0.22 \\
\hline
\end{tabular}

A non-dimensional time step of $1 \times 10^{-5}$ was used for both the cases to satisfy the Courant-Friedrich-Lewy (CFL) stability condition with maximum CFL number of 0.71 . It took approximately 90 hours on 1152 and $1440 \mathrm{CPU}$ cores for one rotation of the cavity for $R a=0.76 \times 10^{8}$ and $1.54 \times 10^{8}$, respectively. For each case, the statistics were collected for approximately 12 rotations.

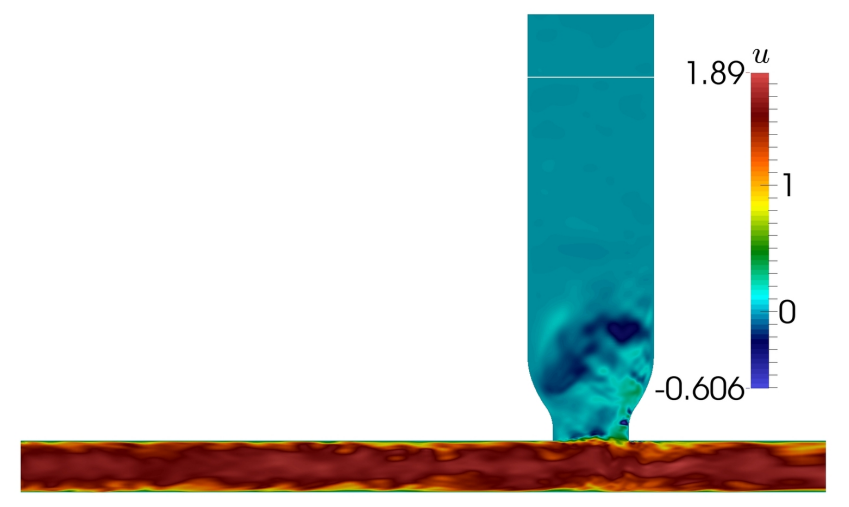

FIGURE 8: Instantaneous axial velocity contour for the cavity showing the turbulent bore flow interaction with the cavity.

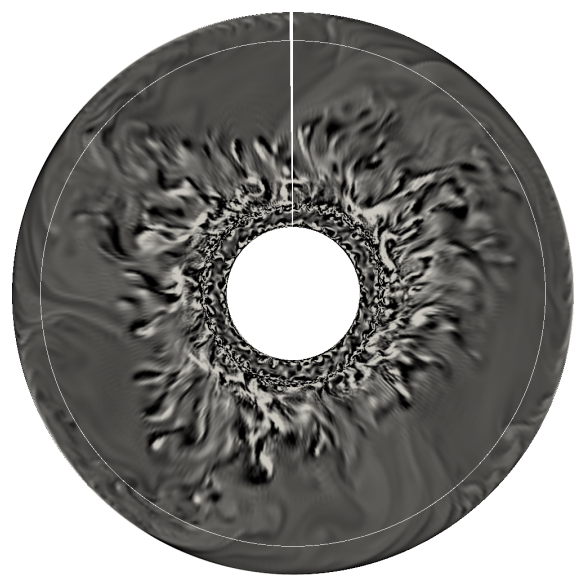

FIGURE 9: Instantaneous contour for circumferential vorticity in the cavity for $R a=1.54 \times 10^{8}$.

\section{Flow Structure Instantaneous Flow Field}

In figure 8 , contours of instantaneous axial velocity illustrate the fully developed turbulent bore flow is entering into the cavity and forming a shear layer at the interface between the bore flow and the outer cavity. The axial bore flow expands into the cavity to form a strong toroidal vortex in the inner region of the cavity shown in figure 9 . This toroidal vortex further bifurcates to radial flares in the outer region of the cavity. These radial flares form cyclonic and anti-cyclonic vortices, which rotate in the direction of rotation and in the opposite direction of rotation of the cavity, respectively. These vortices are responsible for transferring cold fluid from the inner region and hot fluid from the outer region of the cavity. Further, near the shroud, small scale vortices can be observed in figure 9, which corresponds to the presence of local convection cells analogous to Rayleigh-Bénard convection plumes arising due to the centrifugal buoyancy. Figures 10 (a) and 10 (b) show the instantaneous contours of temperature at the mid-axial position of the cavity. The bore flow conditions are the same in both cases. The small scale turbulent structures can be observed in the toroidal vortex region for both cases. However, in the outer region, large scale coherent structures are present. 
(a)
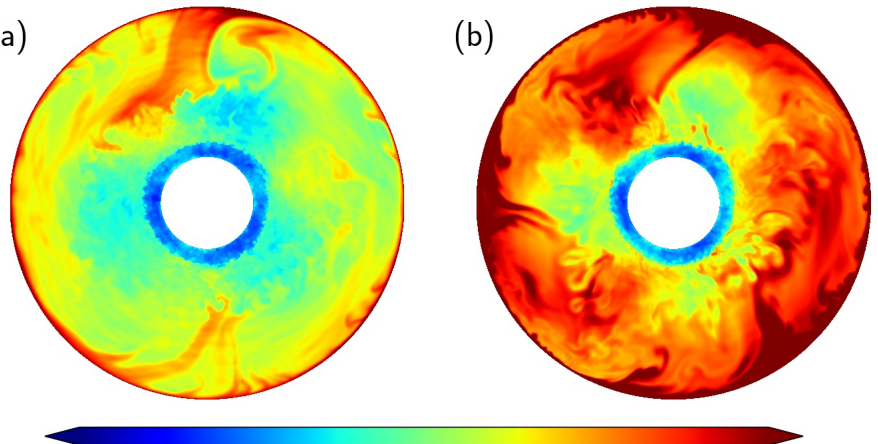

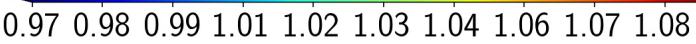
T

(c)

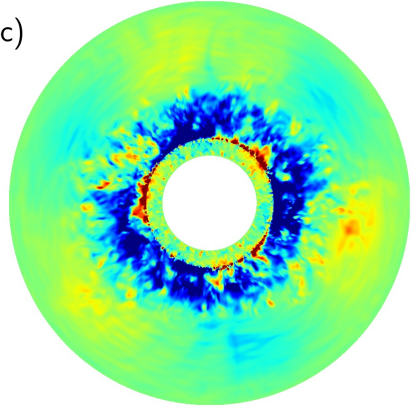

(d)

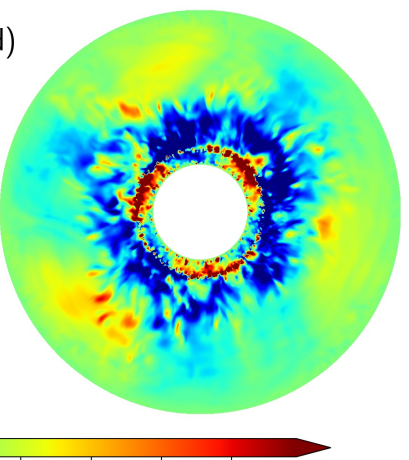

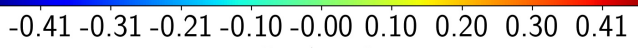
$[v / \Omega r]$

(e)

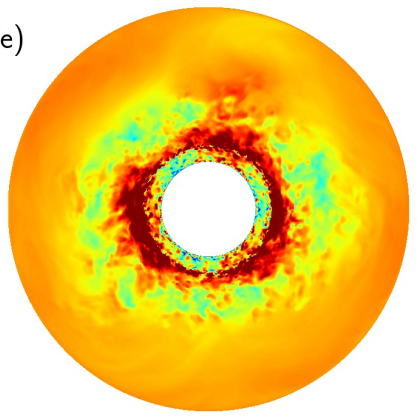

(f)

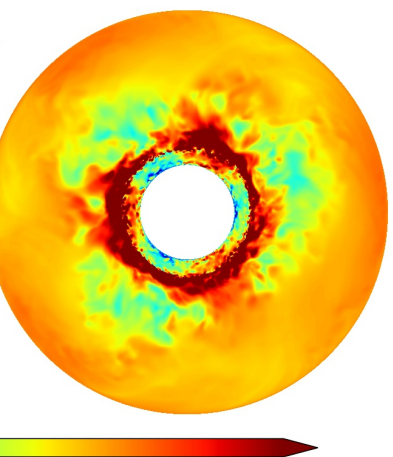

$\begin{array}{llllllllll}-0.41 & -0.20 & 0.00 & 0.21 & 0.41 & 0.61 & 0.82 & 1.02 & 1.23 & 1.43\end{array}$ $[w / \Omega r]$

FIGURE 10: Comparison of instantaneous contours of temperature at (a) $R a=0.76 \times 10^{8}$ and (b) $R a=1.54 \times 10^{8}$, radial velocity scaled with local disk speed at (c) $R a=0.76 \times 10^{8}$ and (d) $R a=1.54 \times 10^{8}$ and circumferential velocity scaled with local disk speed at (e) $R a=0.76 \times 10^{8}$ and (f) $R a=1.54 \times 10^{8}$ at mid-axial position of the cavity rotating in the clockwise direction.

There are three dominant structures in the outer region for both the $R a$ numbers. In figure 10 (a), there is one large structure that spans from the 7'o clock to the 11'o clock positions. A second flow structure extends from the 1'o clock to approximately the 3'o clock position and is followed by a third dominant structure in the outer region. Similarly, three coherent structures can be seen for the high $R a$ number as well in figure 10 (b). For high $R a$ number cases, relatively small scale structures can be observed due to the strong centrifugal buoyant force. Further, to quantify the number of large scale structures, we can also refer to wavenumber spectra shown in figure 7. The spectra show a peak in the energy at a wavenumber $k=3$ for all the monitor points beyond $r=0.5$, which confirms the earlier visual observation made from the instantaneous field. Furthermore, small scale convection plumes can be seen near the shroud region at the 10'o clock position in figure 10 (a) and 12'o clock and 3'o clock positions in figure $10(\mathrm{~b})$, respectively.

The instantaneous contours for the radial velocity and the circumferential velocity scaled with local disk speed are shown in figures $10(\mathrm{c}, \mathrm{d})$ and $10(\mathrm{e}, \mathrm{f})$, respectively. At most of the radial locations, in the toroidal vortex region, the radial velocity is negative. Thus, at the mid-axial location, the flow is directed radially inwards from the cavity. In the outer region, at the three circumferential locations approximately at 4'o clock, 8'o clock and 11'o clock, the radial velocity is positive and in-between these locations radial velocity is negative for both the cases. These three dominant radial flares of positive and negative velocity also correspond to the peak observed in the wavenumber spectra in figure 7. Another quantity of interest is the swirl ratio, defined as the ratio of the circumferential velocity of fluid particles to local disk speed. The swirl ratio contours shown in figure $10(\mathrm{e}, \mathrm{f})$ show the comparison between the two $R a$ numbers. In the toroidal vortex region, the local intensity of swirl is higher for the high $R a$ number case. However, beyond that toroidal vortex region, the values of the swirl ratio reduce to less than one. Thus, fluid particles are rotating slower than the local disk speed and the intensity of this drop increases at high $R a$ number.
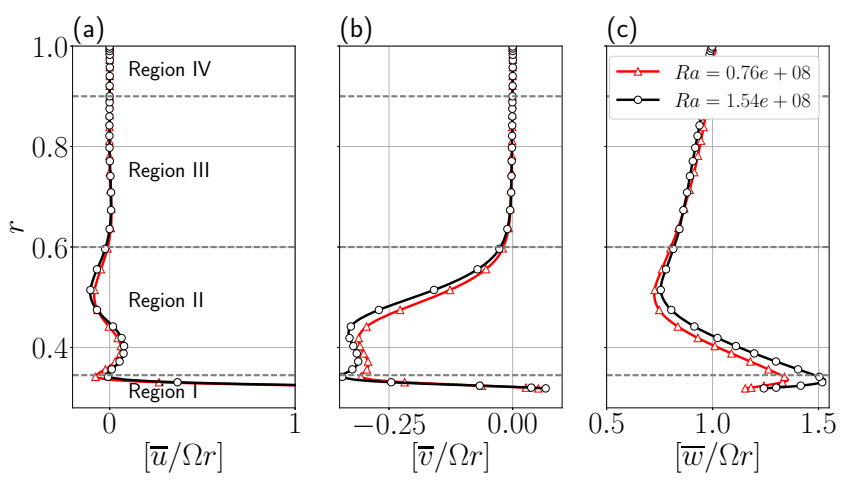

FIGURE 11: Comparison of time and circumferentially averaged velocity profiles at the mid-axial position of the cavity at different Rayleigh numbers.

\section{Mean Statistics}

Although the instantaneous flow field revealed some basic flow features inside the cavity and qualitative comparison between the two $R a$ numbers, thus, to further elucidate the flow structure and quantitative comparison to study the effect of $R a$ number, mean statistics are discussed in this section. Figure 11 shows the velocity profiles averaged over time and the circumferential direction at the mid-axial position of the cavity for different $R a$ numbers. The velocity components are scaled with local disk speed. To understand the mean flow structure, the cavity is divided into four different regions based on the different physical 
(a)

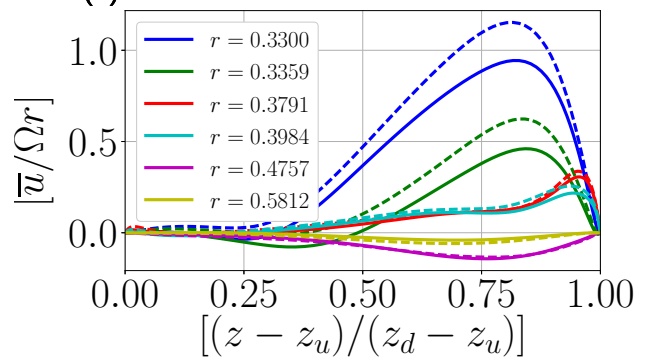

(d)

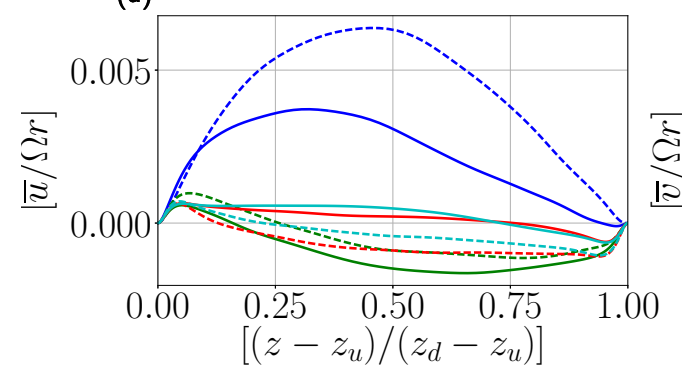

(g)

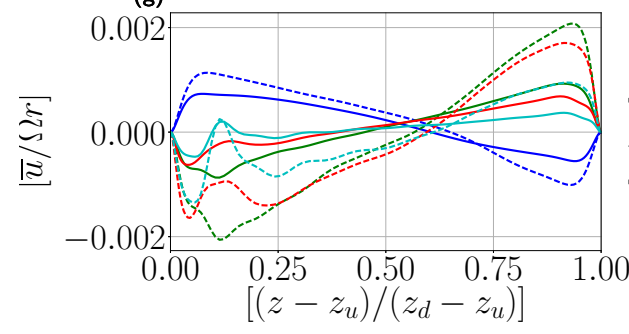

(b)

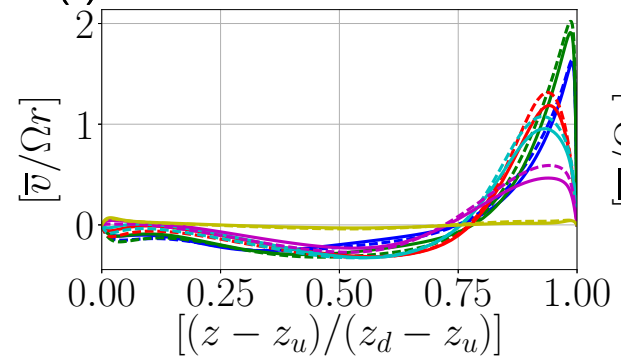

(e)

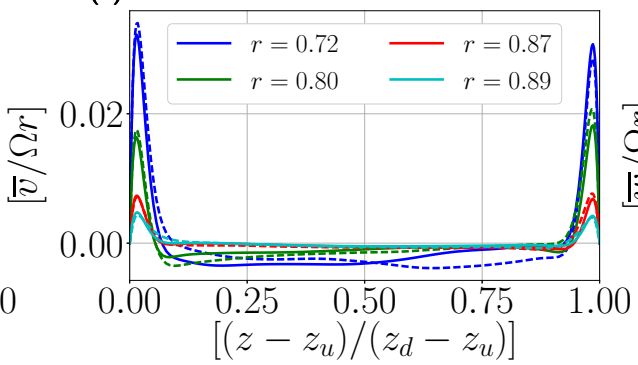

(h)

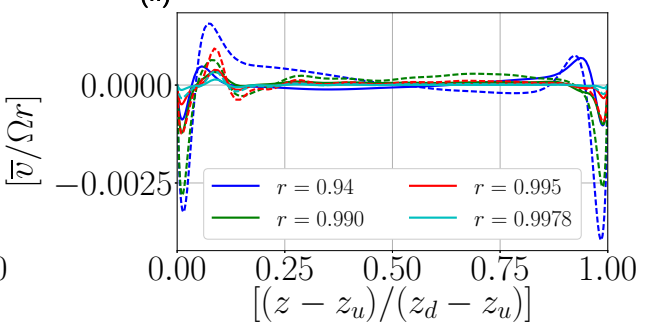

(c)

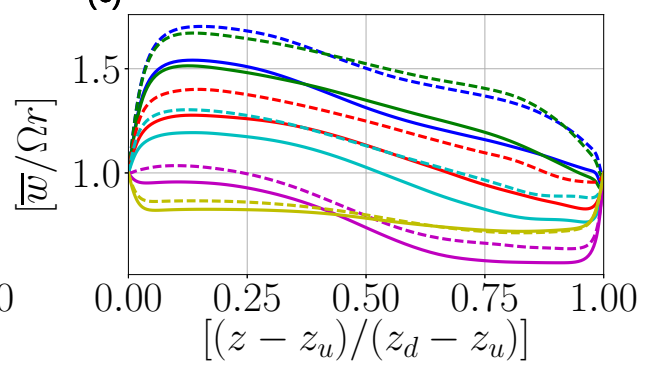

(f)

FIGURE 12: Comparison of time and circumferentially averaged velocity profiles at the different radial locations of the cavity in Region II (a-c), Region III (d-f) and Region IV (g-i) for different Rayleigh numbers $\left(-R a=0.76 \times 10^{8},--R a=1.54 \times 10^{8}\right.$ ).

phenomena observed. The Region I corresponds to an interface between the bore flow and the cavity. A strong shear layer is formed in this region, as shown by the axial velocity profiles in figure 11 (a). There is no perceivable effect of $R a$ number on the velocity profiles in this region as it is dominated by the bore flow conditions, which are same for both the cases.

The bore flow expands into the cavity to form a toroidal vortex in the Region II. The signature of the toroidal vortex can be conceived by the S-shaped profile of the axial velocity in the Region II shown in figure 11 (a), which signifies the flow recirculation due to the vortex ring. There is a subtle change in the magnitude of axial velocity for the higher $R a$ number case as shown in figure 11 (a). However, to analyze the effect of $R a$ number, the variation of axial velocity in the axial direction of the cavity is provided in figure 12 (a). The peak of the axial velocity away from the centre of the cavity is evidence for the asymmetric shape of the toroidal vortex. An overall increase in the axial velocity is observed for the higher $R a$ number case. Further, the S-shape profile of axial velocity at the mid-axial position in figure 11 (a) provides a rough estimate of the radial extent of the toroidal vortex, which is $r \approx 0.6$ for both the $R a$ numbers. The radial extent of the vortex predicted in our study is consistent with the experimental results of Long et al. [9]. They predicted the radial extent of the toroidal vortex for $R o=3.57$ $\left(R e_{\theta}=4.9 e+05, R e_{z}=9.2 e+04\right.$ and $\left.\beta \Delta T_{\text {shroud }}=0.23\right)$ to be approximately $r=0.6$ for the same cavity dimensions as used in our study. Albeit, the value of Ro number was 0.8 times smaller in the experiments; still, there is a good agreement on the extent of the toroidal vortex, which may depend mainly on the cavity dimensions.

Figure 11(b) shows the variation of radial velocity at the mid-axial location of the cavity. The radial velocity is negative, which shows that the flow is directed inwards at this location. The variation of radial velocity in the axial direction in Region II is shown in figure 12 (b). Again, the profiles show the lack of symmetry in the axial direction of the toroidal vortex. The flow direction into the cavity is biased towards the downstream disk of the cavity. The flow enters the cavity by the boundary layers formed at the downstream disk covering $20 \%$ of the axial length for flow ingestion. It leaves the cavity through the remaining $80 \%$ of the cavity opening via the upstream disk. The comparison of radial velocity profiles for the two $R a$ numbers shows a small increase in the magnitude of radial velocity for the higher $R a$ number case. Overall, a 7\% enhancement in the mass flow rate from the bore flow into the cavity is observed for the higher $R a$ number case. Furthermore, an additional $7.2 \%$ pressure loss from the bore flow inlet to the outlet is observed in the case of higher $R a$ number. We speculate that the high swirl ratio in the lower radii of the cavity could be the potential cause of this pressure loss in the bore flow passage, which is detrimental to the overall performance of the internal air system [23]. The radial variation of swirl ratio, which directly affects the pressure loss, 

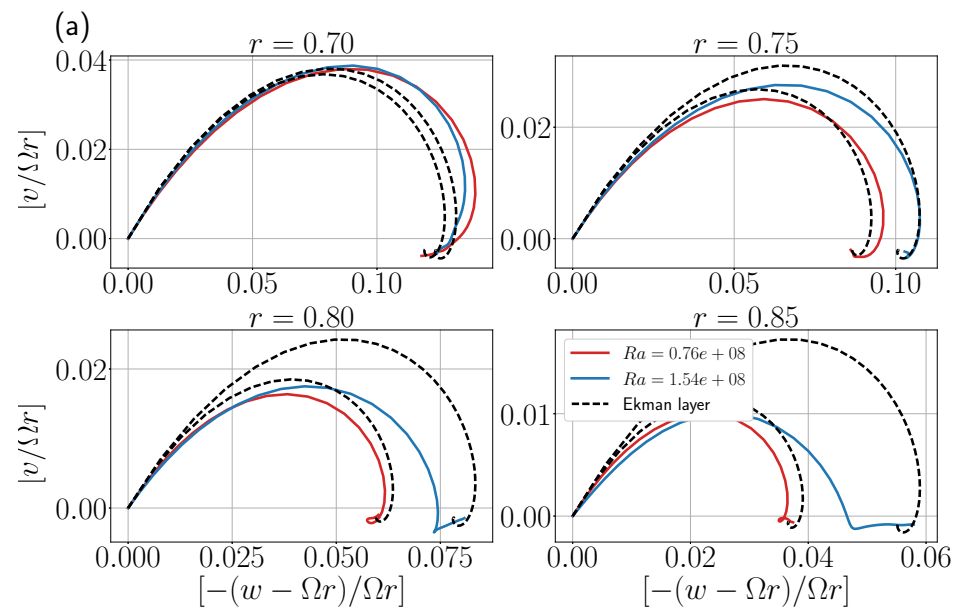

(b)

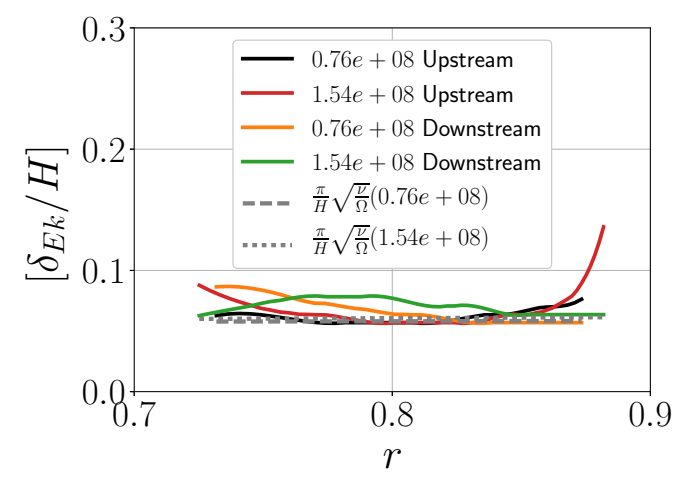

FIGURE 13: (a) Ekman spiral formed at different radial locations on upstream disk and comparison with the solution of linear Ekman layer equations, (b) Comparison of boundary layer thickness for different $R a$ numbers on both the disks scaled with disk gap $\mathrm{H}$.

is shown in figure 11(c). The peak value of swirl ratio is close to 1.5 for $R a=1.54 e+08$ and reduces to approximately 1.3 for the low $R a$ number case. The peak location of swirl ratio $r \approx 0.34$ is also found to be consistent with the experimental results of [9] for the same cavity dimensions. The axial variation of the swirl ratio in Region II is shown in figure 12 (c). The peak of the swirl ratio lies near the upstream disk, and a significant effect of $R a$ number on the swirl ratio is perceived at the cavity inlet. The rise in the swirl ratio signifies the high intensity or strength of the toroidal vortex at the higher $R a$ number. In addition, the rise in the mass flow rate at the higher $R a$ number may also be related to the strength of the toroidal vortex, as the centrifugal buoyancy has minimal effect in this region. Thus, it is important to study the effect of various parameters on the vortex strength, which could be the reason behind the increase in the swirl ratio and the mass flow rate into the cavity. However, this is beyond the scope of this paper.

The Region III of the cavity, away from the toroidal vortex is less energetic and dominated by the centrifugal-buoyant force. It has radial flares emerging from the toroidal vortex moving radially outwards as well as inwards in the cavity. The magnitude of axial velocity in this region is very small, as shown in figure 12(d). Thus, the flow is mostly directed towards the radial and the circumferential directions. The axial variation of the radial velocity in Region III shows that the flow moves radially outwards through the boundary layers formed on the side disk and inwards by the central core of the cavity, as shown in figure 12 (e). The boundary layers formed on the side disks are threedimensional in nature and called Ekman layers. In the Ekman layer at a particular radial location on the side disk, the flow in the core of the cavity is aligned in the circumferential direction and near to the wall turns in the radial direction forming an Ekman spiral, as shown in figure 13 (a). The velocity components plotted in figure 13 (a) are in the frame of reference of rotation. The Ekman spiral shows the direction of the resultant velocity vector, which gradually changes from the radial to circumferential direction in the wall-normal direction. The distance in the wall-normal direction at which the radial or transverse velocity component crosses the origin is defined as a boundary layer thickness [24].

The Ekman spiral formed on the side disk is compared with the solution of linear Ekman layer equations [24] (equation 9 in the appendix) by assuming the flow to be steady, laminar and that the Coriolis force term is much larger than the nonlinear inertial terms in the equations. The comparison shows a reasonable agreement at $r=0.70$ and 0.75 . However, at $r=0.85$ and 0.89 , the shape of the Ekman spiral is different than the prediction of linear Ekman layer equations. The peak radial velocity has reduced at both the radial locations. However, there is good agreement in terms of Ekman depth, where radial velocity crosses the origin. Furthermore, the solution of linear Ekman layer equations [24] provides the boundary layer thickness

$$
\delta_{E k}=\pi \sqrt{\frac{\mu}{\rho \Omega}},
$$

at which the transverse velocity component crosses the origin. Figure 13 (b) shows the comparison of the Ekman layer thickness at both the side disks for both $R a$ numbers. A quasiconstant boundary layer thickness is observed for both the $R a$ numbers, except at the downstream disk for the higher $R a$ number case for which the boundary layer thickness reduces in the radial direction. The relative comparison of boundary layer thickness between the two $R a$ number shows a rise with the $R a$ number. Thus, at higher $R a$ number, the radial flow rate inside the Ekman layers towards the shroud increases. The comparison with the analytical result in equation 7 shows a good agreement for low $R a$ number case at both upstream and downstream disks. However, for the high $R a$ number case, the Ekman boundary layer thickness predicted from our simulations is higher than the analytical result. One possible reason for these differences could be the rise in the level of root-mean-square of radial velocity $v_{r m s}$ fluctuations, and it defies the laminar assumption used to derive equation 7. For instance, the comparison of $v_{r m s}$ at $r=0.8$ for both $R a$ numbers integrated over wall-normal distance $\delta_{E k}$ shows the two times stronger fluctuations at higher $R a$ number. Further, the comparison of the near-wall maximum value of $v_{r m s}$ with the radial velocity $v$ shows order-of-magnitude smaller values of fluctuations. This result is in contrast with the closed cavity results as reported by Pitz et al. [25] for which $v_{r m s}$ values were order-of-magnitude larger than the radial velocity itself. Thus, they defined the boundary layer thickness based on the maximum value of $v_{r m s}$. However, in our study, the relatively smaller 
(a)
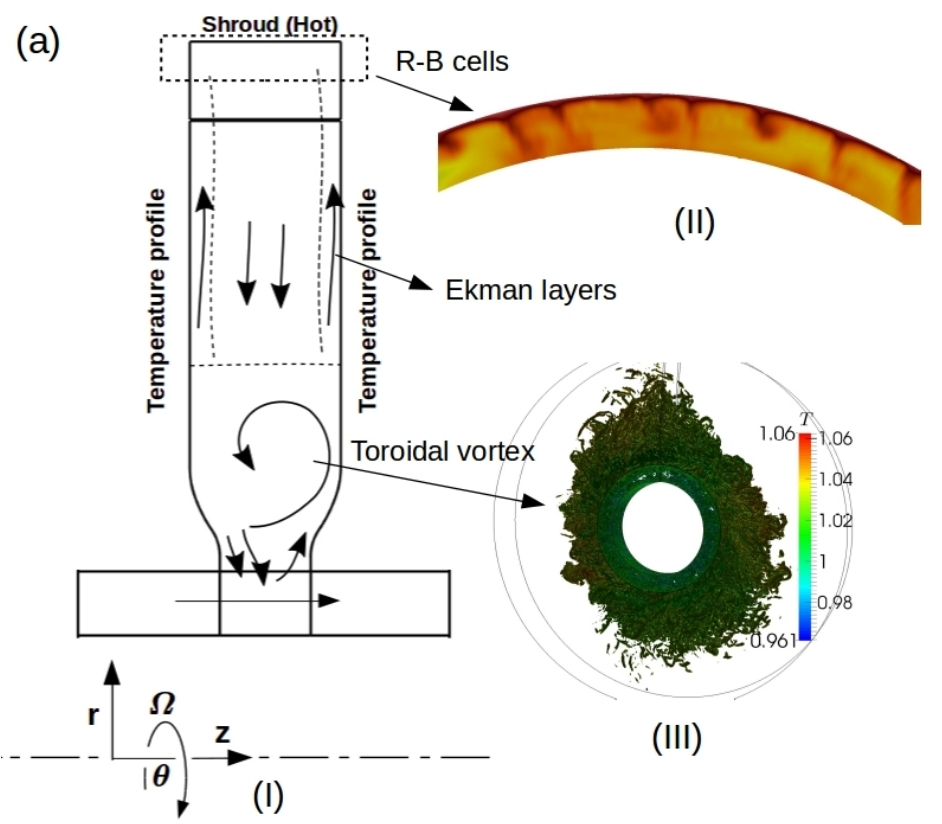

(III)

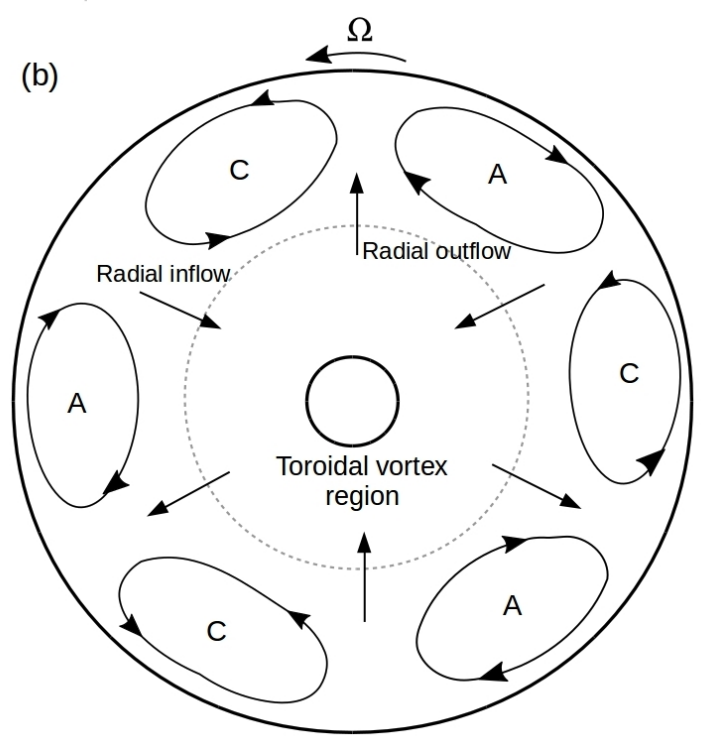

FIGURE 14: (a) Schematic of flow structure for the heated shroud and side disks with radially increasing temperature profile (I) interpretation from the time and circumferentially averaged statistics, (II) instantaneous temperature contours near the shroud shows the local convection cells (III) visualisation of toroidal vortex by iso-surfaces of $\lambda$-ci colored by local temperature, (b) Flow structure in the radial-circumferential plane interpreted from the instantaneous flow field, where $\mathrm{C}$ refers to cyclonic and A to anti-cyclonic vortices.

magnitude of radial velocity fluctuations with respect to velocity leads to good agreement with the laminar Ekman layer thickness defined by equation 7 , as shown in figure 13 (b). Thus, the solution to laminar Ekman layer equations and the derived closed-form equation 7 for boundary layer thickness is a good approximation.

Further, the variation of swirl ratio in the axial direction in Region III is shown in figure 12 (f). It reduces to a value of less than one in the outer region. Thus, the flow is rotating slower than the local disk speed, and it further reduces with the in-

crease in the $R a$ number and temperature of the side disks. This phenomenon was also observed in the experiments by Owen et al. [5]. They observed a decrease in the value of local swirl ratio with a rise in the temperature of the side disk (only one of the disks was heated in experiments). However, as we move along the radial direction, the local circumferential velocity approaches the local disk speed. The Region IV is mostly dominated by small scale convection cells formed due to the centrifugal buoyancy. Figure 12 (g-i) show the averaged velocity profiles in this region. The radial velocity is negative in the disk boundary layers, which shows that the flow is directed towards the toroidal vortex. In the core region, the radial velocity is positive, which infers the formation of local convection plumes, which rise towards the shroud due to the centrifugal buoyancy. The relative comparison between the two $R a$ numbers shows a significant increase in the magnitude of radial velocity due to the large buoyant force for the higher $R a$ number case.

The salient features of the flow structure, as discussed above, have been summarised in figure 14. The axial bore flow expands into the cavity and forms a toroidal vortex, which evolves with time. A fluid particle in the toroidal vortex region follows a helical path. The curvature of the cobs at the cavity inlet induce the vorticity in the $r-z$ plane, which extends in the circumferential direction to form a closed loop and rotates with the cavity walls to form the toroidal vortex. The asymmetric toroidal vortex leads to a flow structure biased towards the downstream disk and forms a boundary layer through which the flow enters into the cavity. This physical phenomenon is identical to the one observed by Farthing et al. [2], shown in figure 2. The radial flares emanating from the toroidal vortex move towards the shroud due to the centrifugal buoyancy. They form the Ekman layer on the side disks and flow moves radially outwards towards the shroud and moves radially inwards by the core region of the cavity as concluded from the time and circumferentially averaged statistics. These results are contrary to the flow structure observed by [2] for a radially decreasing temperature distribution on the side disks. The interpretation of the instantaneous flow field in the radial and circumferential plane is shown in figure 14 (b). The toroidal vortex bifurcates to form three dominant radial flares in the outer region of the cavity. The radial flares moving outwards create a pair of cyclonic and anti-cyclonic vortices. The flow between the consecutive anti-cyclonic and cyclonic vortices created by two different radial outward moving flares is being pushed towards the toroidal vortex, as shown in figure 14 (b). In the near-shroud region, the vigour of the radial flares reduces or is limited to a few circumferential locations. Thus the outer region is mostly dominated by the local convection cells induced by the centrifugal buoyancy force, as shown by instantaneous temperature contours in figure 14 (a-II).

\section{Heat Transfer}

In this section, the results of wall heat transfer rate from the side disks and the shroud are presented. Figure 15 shows the mean density and temperature profile in each region of the cavity. The mean density and temperature profiles in the Region II (figures 15 (a) and (b)) of the cavity show the conditions which lead to negative heat transfer rate from the fluid to the side disk. The mixing of hot fluid from the outer region of the cavity with 
the bore flow leads to a rise in the average temperature of the fluid in this region compared to the temperature of the side disks. The density of the fluid also drops due to this thermal mixing of hot and cold fluid.

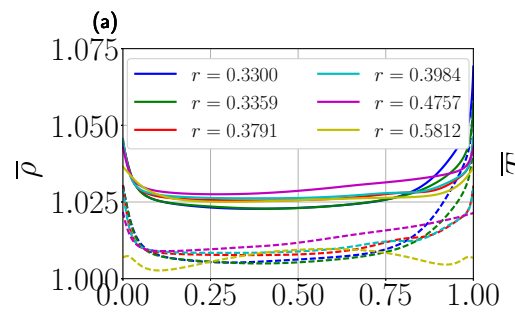

(c)
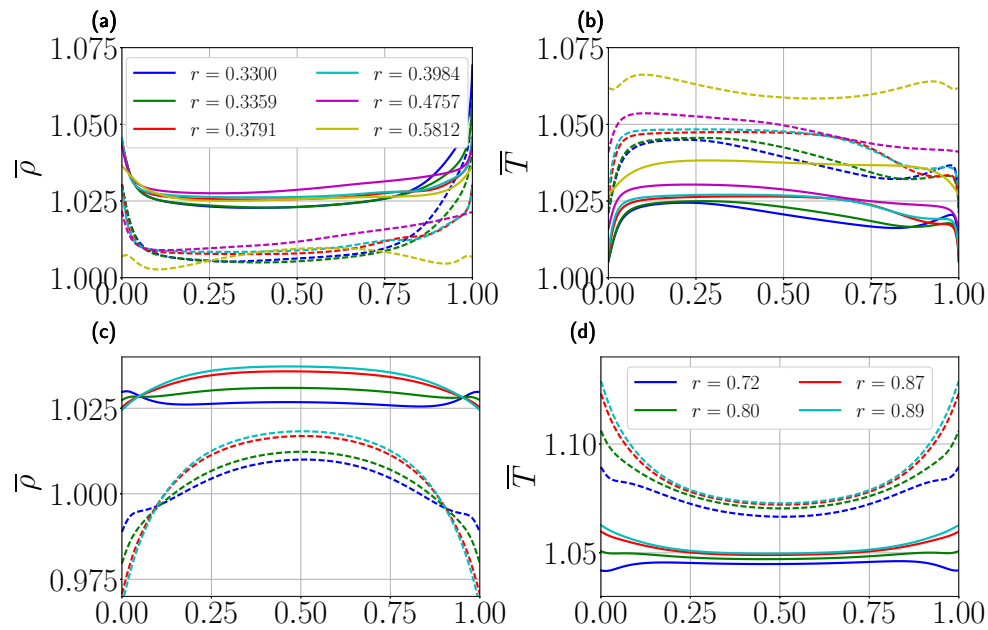

(d)
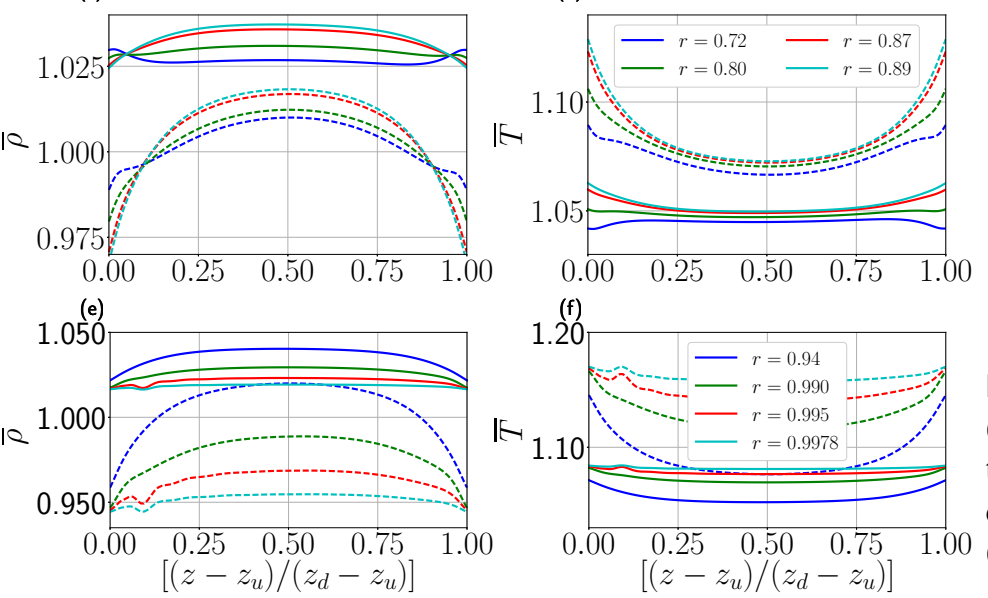

(a)

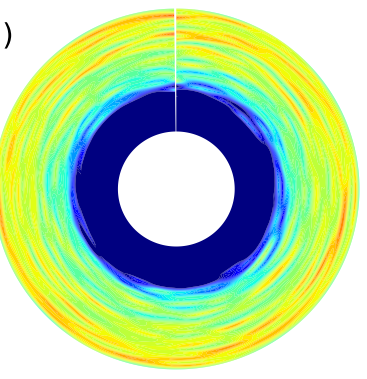

(c)

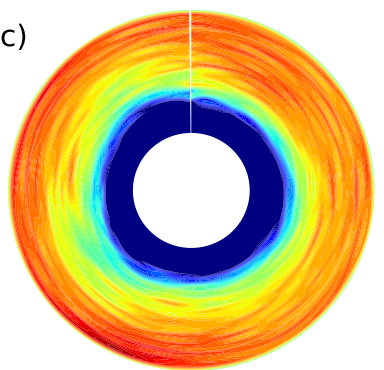

(d)
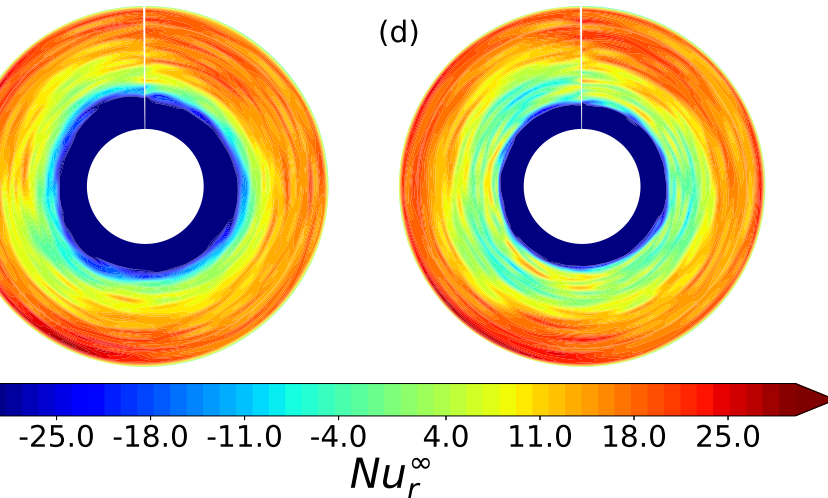

(b)

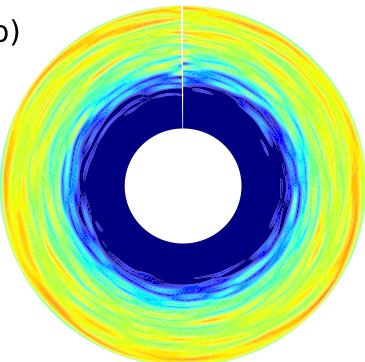

FIGURE 16: Comparison of time averaged Nusselt number $\left(N u_{r}^{\infty}=\left[r /\left(T_{w}-T_{\infty}\right)\right][-d \bar{T} / d z]_{w}\right)$ calculated based on the shaft temperature $\left(T_{\infty}\right)$ for $R a=0.76 \times 10^{8}$ at (a) upstream disk, (b) downstream disk and for $R a=1.54 \times 10^{8}$ at (c) upstream disk,

(d) downstream disk.
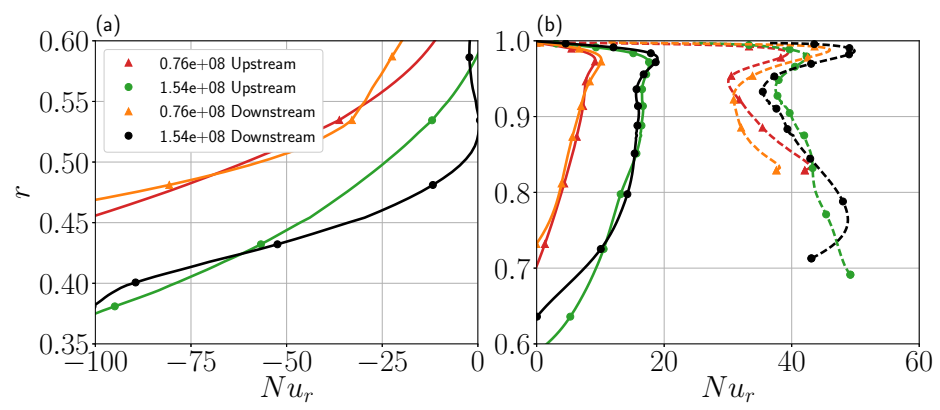

FIGURE 17: Comparison of the variation of local Nusselt number defined based on the shaft temperature $\left(T_{\infty}\right)\left(-N u_{r}^{\infty}=\right.$ $\left.\left[r /\left(T_{w}-T_{\infty}\right)\right][-d \bar{T} / d z]_{w}\right)$ and based on the core cavity temperature $\left(--N u_{r}^{c}=\left[r /\left(T_{w}-T_{c}\right)\right][-d \bar{T} / d z]_{w}\right)$ on the side disks for different Rayleigh numbers.

pared to $r=0.7$ for the low $R a$ number case. Overall, a higher heat transfer rate is observed for $R a=1.54 e+08$ at both the disks as compared to the low $R a$ number case. The distribution of $N u_{r}^{\infty}$ is quasi-linear without much variation between the two disks. However, the Nusselt number $N u_{r}^{c}$ calculated based on the local core temperature in the cavity provides the more sensible quantification of local heat transfer effectiveness. It should be noted, though, that this definition breaks down at the radial location where the core temperature reaches values close to the local disk temperature and the denominator in equation 6 therefore approaches zero. The comparison of $N u_{r}^{c}$ on the side disks for both the $R a$ numbers shows higher values at the downstream disk as compared to the upstream one, and the heat transfer rate is fur- 


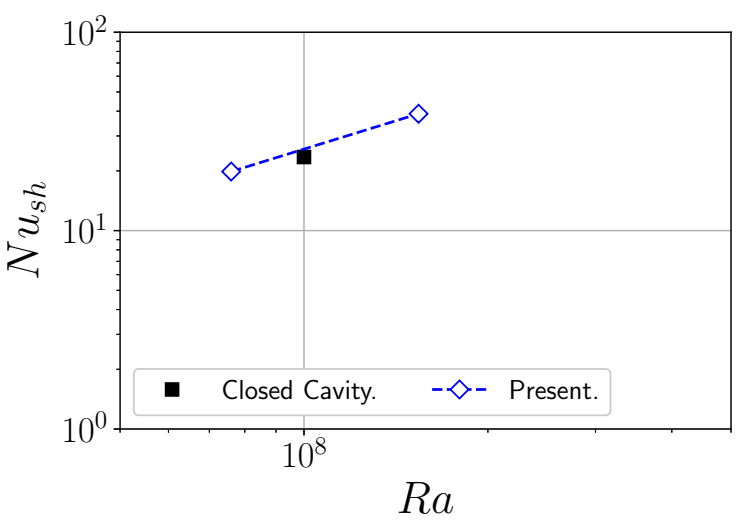

FIGURE 18: Comparison of shroud Nusselt number with the results of closed cavity without bore flow from [20] .

ther enhanced for the higher $R a$ number case. In the near-shroud region, the Nusselt number approaches zero as the temperature gradient in the axial direction tends to zero due to the same temperature level of the side disks and the shroud.

Further, the shroud $\mathrm{Nu}$ number is calculated based on the definition used for a closed cavity [20]

$$
N u_{s h}=\frac{q_{\text {convection }}}{q_{\text {conduction }}}=\frac{\ln \left(r_{o} / r_{i}\right)}{\Delta T / T_{\infty}}\left[\frac{\mathrm{d} \overline{\mathrm{T}}}{\mathrm{d} r}\right]_{r=1},
$$

where $\Delta T=T_{s h}-T_{\infty}$. The $N u$ numbers calculated for both $R a$ numbers are shown in figure 18. The comparison with the $N u$ number predicted for a closed cavity without the bore flow for $R a=10^{8}$ [20] is also shown in figure 18. All the values of $N u$ numbers lie on a straight line, which confirms that the centrifugal buoyancy-induced flow governs the heat transfer in the near shroud region and the axial bore flow does not affect it.

\section{Conclusions}

In this study, we conducted highly-resolved numerical simulations for flow in a high-pressure compressor inter-disk cavity for two $R a$ numbers of $0.76 \times 10^{8}$ and $1.54 \times 10^{8}$ at a fixed $R o$ number of 4.5 , matching the experimental conditions of Atkins and Kanjirakkad [11]. The results of the present study show that the axial bore flow expands into the cavity to form a toroidal vortex. The toroidal vortex formed in the inner region has a radial extent up to $r \approx 0.6$, and the change in $R a$ number shows no effect on the radial extent. An increase in the radial mass flow rate and swirl ratio was observed for higher $R a$ number case. For both the $R a$ numbers, three dominant structures in the outer region of the cavity, away from the toroidal vortex, were observed which correspond to three pairs of cyclonic and anti-cyclonic vortices. In this outer region, Ekman layers form on the side disk and were responsible for radial flow towards the shroud. The thickness of the Ekman layers increases with the increase in the $R a$ number. Further, the thickness of the Ekman layers was found to be in good agreement with the solution of linear Ekman layer equations for the low $R a$ number case and deviated for the high $R a$ number. The mixing of hot fluid from the outer region with the cold bore flow leads to a rise in the average temperature of the flow in the toroidal vortex region and a negative heat transfer rate to the side disks was observed for both the $R a$ numbers. The flow features near the shroud region were reminiscent of RayleighBénard streaks evolved in centrifugal-buoyant situations. The averaged wall $\mathrm{Nu}$ number at the shroud for both the $R a$ numbers and the $N u$ number for a closed cavity without bore flow were shown to all lie on a straight line, which highlights the inefficacy of the bore flow to enhance the shroud heat transfer rate.

\section{ACKNOWLEDGMENT}

This work was supported by resources provided by the Pawsey Supercomputing Centre with funding from the Australian Government and the Government of Western Australia through the National Computational Merit Allocation Scheme. We would like to thank Antonio Guijarro for various stimulating discussions.

\section{REFERENCES}

[1] Epstein, A. H., 2014. "Aeropropulsion for commercial aviation in the twenty-first century and research directions needed". AIAA journal, 52(5), pp. 901-911.

[2] Farthing, P., Long, C., Owen, J., and Pincombe, J., 1992. "Rotating cavity with axial throughflow of cooling air: flow structure". ASME J. Turbomach, 114(1), pp. 237-246.

[3] Farthing, P., Long, C., Owen, J., and Pincombe, J., 1992. "Rotating cavity with axial throughflow of cooling air: heat transfer". ASME J. Turbomach, 114(1), pp. 229-236.

[4] Bohn, D. E., Deutsch, G. N., Simon, B., and Burkhardt, C., 2000. "Flow visualisation in a rotating cavity with axial throughflow". ASME paper(2000-GT), p. 280.

[5] Owen, J. M., and Powell, J., 2006. "Buoyancy-induced flow in a heated rotating cavity". Journal of Engineering for Gas Turbines and Power, 128(1), pp. 128-134.

[6] Owen, J. M., Abrahamsson, H., and Lindblad, K., 2007. "Buoyancy-induced flow in open rotating cavities". Journal of Engineering for Gas Turbines and Power, 129(4), pp. 893-900.

[7] Owen, J. M., and Long, C. A., 2015. "Review of buoyancyinduced flow in rotating cavities". Journal of Turbomachinery, 137(11), p. 111001.

[8] Owen, J. M., and Tang, H., 2015. “Theoretical model of buoyancy-induced flow in rotating cavities". Journal of Turbomachinery, 137(11), p. 111005.

[9] Long, C., Miché, N., and Childs, P., 2007. "Flow measurements inside a heated multiple rotating cavity with axial throughflow". International Journal of Heat and Fluid Flow, 28(6), pp. 1391-1404.

[10] Sun, Z., Lindblad, K., Chew, J. W., and Young, C., 2007. "LES and RANS investigations into buoyancy-affected convection in a rotating cavity with a central axial throughflow". Journal of Engineering for Gas Turbines and Power, 129(2), pp. 318-325.

[11] Atkins, N. R., and Kanjirakkad, V., 2014. "Flow in a rotating cavity with axial throughflow at engine representative conditions". In ASME Turbo Expo 2014: Turbine Techni- 
cal Conference and Exposition, American Society of Mechanical Engineers Digital Collection.

[12] Puttock-Brown, M., Rose, M., and Long, C., 2017. "Experimental and computational investigation of rayleigh-bénard flow in the rotating cavities of a core compressor". In ASME Turbo Expo 2017: Turbomachinery Technical Conference and Exposition, American Society of Mechanical Engineers Digital Collection.

[13] Pitz, D. B., Chew, J. W., and Marxen, O., 2019. "Effect of an axial throughflow on buoyancy-induced flow in a rotating cavity". International Journal of Heat and Fluid Flow, 80, p. 108468.

[14] Touber, E., and Sandham, N. D., 2009. "Large-eddy simulation of low-frequency unsteadiness in a turbulent shockinduced separation bubble". Theoretical and Computational Fluid Dynamics, 23(2), pp. 79-107.

[15] Sandberg, R. D., and Sandham, N. D., 2006. "Nonreflecting zonal characteristic boundary condition for direct numerical simulation of aerodynamic sound". AIAA journal, 44(2), pp. 402-405.

[16] Sandberg, R., 2015. "Compressible-flow DNS with application to airfoil noise". Flow, Turbulence and Combustion, 95(2-3), pp. 211-229.

[17] Sandberg, R. D., Michelassi, V., Pichler, R., Chen, L., and Johnstone, R., 2015. "Compressible direct numerical simulation of low-pressure turbines Part I: Methodology". Journal of Turbomachinery, 137(5), p. 051011.

[18] Pichler, R., Sandberg, R. D., Laskowski, G., and Michelassi, V., 2017. "High-fidelity simulations of a linear hpt vane cascade subject to varying inlet turbulence". In ASME Turbo Expo 2017: Turbomachinery Technical Conference and Exposition, American Society of Mechanical Engineers Digital Collection.

[19] Leggett, J., Priebe, S., Shabbir, A., Michelassi, V., Sandberg, R., and Richardson, E., 2018. "Loss prediction in an axial compressor cascade at off-design incidences with free stream disturbances using large eddy simulation". Journal of Turbomachinery, 140(7), p. 071005.

[20] Saini, D., Chung, D., and Sandberg, R. D., 2018. "Direct numerical simulations of centrifugal buoyancy induced flow in a closed rotating cavity". In Proceedings of 21 st Australasian Fluid Mechanics Conference, Adelaide, Australia (2018).

[21] Saini, D., and Sandberg, R. D., 2020. "Simulations of compressibility effects in centrifugal buoyancy-induced flow in a closed rotating cavity". International Journal of Heat and Fluid Flow, 85, p. 108656.

[22] Nicoud, F., and Ducros, F., 1999. "Subgrid-scale stress modelling based on the square of the velocity gradient tensor". Flow, Turbulence and Combustion, 62(3), pp. 183200.

[23] Pfitzner, M., and Waschka, W., 2000. "Development of an aeroengine secondary air system employing vortex reducers". In 22nd ICAS Congress, Harogate, UK, Aug, pp. 5111.

[24] Childs, P. R., 2010. Rotating flow. Elsevier.

[25] Pitz, D. B., Chew, J. W., and Marxen, O., 2019. "Largeeddy simulation of buoyancy-induced flow in a sealed rotating cavity". Journal of Engineering for Gas Turbines and Power, 141(2), p. 021020.

\section{Appendix Temperature distribution on side disks}

TABLE 2: Non-dimensional side disk temperature with respect to $T_{\infty}$ for case I and II

\begin{tabular}{c|c|c}
\hline \hline$r$ & Case I & Case II \\
\hline \hline 0.3187 & 1.0049 & 1.0208 \\
0.3772 & 1.0075 & 1.0251 \\
0.4357 & 1.0120 & 1.0336 \\
0.4943 & 1.0177 & 1.0443 \\
0.5528 & 1.0239 & 1.0559 \\
0.6113 & 1.0302 & 1.0678 \\
0.6698 & 1.0365 & 1.0795 \\
0.7284 & 1.0427 & 1.0911 \\
0.7869 & 1.0492 & 1.1032 \\
0.8454 & 1.0564 & 1.1168 \\
0.9000 & 1.0643 & 1.1319 \\
0.9202 & 1.0677 & 1.1384 \\
0.9404 & 1.0714 & 1.1455 \\
0.9606 & 1.0754 & 1.1534 \\
0.9808 & 1.0799 & 1.1620 \\
1.0000 & 1.0846 & 1.1712 \\
\hline \hline
\end{tabular}

\section{Solution to linear Ekman layer equations}

The final solution to the Ekman layer equations [24] [25] is

$$
\begin{aligned}
& v=v_{\text {core }}\left[1-e^{-z / \delta} \cos (z / \delta)\right]-w_{\text {core }} e^{-z / \delta} \sin (z / \delta), \\
& w=w_{\text {core }}\left[1-e^{-z / \delta} \cos (z / \delta)\right]+v_{\text {core }} e^{-z / \delta} \sin (z / \delta),
\end{aligned}
$$

where, $\delta=\sqrt{\mu /(\rho \Omega)}$, defined as the Ekman depth. The $v_{\text {core }}$ and $w_{\text {core }}$ are the axially averaged core radial and the circumferential velocity components, respectively. 


\section{University Library}

\section{- M M N E R VA A gateway to Melbourne's research publications}

Minerva Access is the Institutional Repository of The University of Melbourne

Author/s:

Saini, D;Sandberg, R

Title:

LARGE EDDY SIMULATIONS OF HIGH ROSSBY NUMBER FLOW IN THE HIGH PRESSURE COMPRESSOR INTER-DISK CAVITY

Date:

2021

Citation:

Saini, D. \& Sandberg, R. (2021). LARGE EDDY SIMULATIONS OF HIGH ROSSBY NUMBER FLOW IN THE HIGH PRESSURE COMPRESSOR INTER-DISK CAVITY. Proceedings of ASME Turbo Expo 2020 Turbomachinery Technical Conference and Exposition GT2020, 7C-2020, ASME: The American Society of Mechanical Engineers. https://doi.org/10.1115/ GT2020-14463.

Persistent Link:

http://hdl.handle.net/11343/242436 